International Journal of Automotive and Mechanical Engineering (IJAME)

ISSN: 2229-8649 (Print); ISSN: 2180-1606 (Online); Volume 7, pp. 1086-1095, January-June 2013

(C)Universiti Malaysia Pahang

DOI: http://dx.doi.org/10.15282/ijame.7.2012.23.0088

\title{
SOUND TRANSMISSION LOSS OF A DOUBLE-LEAF PARTITION WITH MICRO-PERFORATED PLATE INSERTION UNDER DIFFUSE FIELD INCIDENCE
}

\author{
A. Putra ${ }^{*}$, A. Y. Ismail and Md. R. Ayob \\ Faculty of Mechanical Engineering \\ Universiti Teknikal Malaysia Melaka (UTeM) \\ Hang Tuah Jaya, 76100, Melaka, Malaysia \\ Email: azma.putra@utem.edu.my
}

\begin{abstract}
In noise control applications, a double-leaf partition has been applied widely as a lightweight structure for noise insulation, such as in car doors, train bodies, and aircraft fuselages. Unfortunately, the insulation performance deteriorates significantly at massair-mass resonance due to coupling between the panels and the air in the gap. This paper investigates the effect of a micro-perforated panel (MPP), inserted in the conventional double-panel partition, on sound transmission loss at troublesome resonant frequencies. It is found that the transmission loss improves at this resonance if the MPP is located at a distance of less than half that of the air gap. A mathematical model is derived for the diffuse field incidence of acoustic loading.
\end{abstract}

Keywords: Transmission loss; double-panel; noise; MPP; acoustics.

\section{INTRODUCTION}

A double-leaf structure is a common structural design in many engineering applications. Body structures of cars, trains, and airplanes, as well as the walls of buildings are some examples of double-leaf partitions in practice. The double-leaf has been found to be a better noise barrier compared with the single-leaf. However, there remains a problem with the double-panel, which is the weak sound transmission loss (STL) performance at low frequencies due to the "mass-air-mass" resonance. At this frequency range, this causes the double-leaf to lose its superiority over the single-leaf structure (Fahy and Gardonio, 2006). Several works have been published on attempts to overcome this problem. This includes the application of absorptive materials inside the gap of a double-leaf to increase damping (Tang et al., 1998, Bravo et al., 2002). This treatment was found to be effective in increase STL at low frequency, including that of the massair-mass resonance. Another work suggested installing Hemholtz resonators in the air gap (Mao and Piertzko, 2005). This resonator acts like a single-degree of freedom system, the natural frequency of which depends on its geometry. It is found that by optimally tuning the resonator, the STL at resonance is improved significantly.

Some active noise control systems have also been proposed to solve the massair-mass resonance problem. Two different pieces of equipment, i.e., a loudspeaker and an actuator were placed inside the gap to control actively the sound transmission ( $\mathrm{Li}$ and Cheng, 2008). The loudspeaker reduces the transmission energy that propagates through the acoustic path, and the actuator reduces energy from the structural path by creating a counter force on the two panels to suppress the vibration; this in turn reduces the radiated sound into the air. By varying the distance between both the loudspeaker and 
the actuator to the panels, both strategies gave effective reduction of sound transmission. Similarly, a long T-shaped resonator was embedded along the edge of the double panel to control actively the acoustic path inside the gap (Li et al., 2010). This was also been found effective in increasing STL at an even broader frequency range.

In 1975, Dah You Maa in China found that when a thin panel with submillimeter-sized holes was introduced and located in front of a rigid panel with an air gap, the micro-perforated panel (MPP) acts as a sound absorber following a Helmholtz resonator mechanism. The diameter of the holes must be within the range $0.05-1 \mathrm{~mm}$ and the perforation ratio must be between $0.5 \%$ and $1.5 \%$ for optimum absorption (Maa, 1975). As the MPP is made from a panel, it provides several advantages, such as being non-fibrous, non-abrasive, and non-polluting. Several works have been conducted concerning the improvement of sound absorption performance of the MPP, e.g., by arranging it to be a double-leaf (Sakagami et al., 2006), by increasing its thickness (Pfretzner and Cobo, 2006), and by modifying its hole geometry (Sakagami et al., 2008). This paper proposes the insertion of an MPP within a double-panel partition. The proposed system might be important for a condition where any abrasive and toxic material, such as commercial glass wool and glass fiber cannot be presented for health reasons. The next section describes the derivation of the mathematical model and the simulation results that show the effect of the MPP insertion, in terms of the location of the MPP in the gap, as well as its hole size and perforation ratio on the performance of the sound transmission loss, particularly at the mass-air-mass resonance.

\section{GOVERNING EQUATIONS}

\section{Propagating Acoustic Pressure}

Figure 1 shows a mechanical system consisting of double solid panels with an MPP inserted in between (abbreviated here as DL-MPP), which is excited by an oblique incidence of sound with an arbitrary angle $\varphi$. The solid panels are separated by distance $D$ and the MPP is located at distance $l$ from the back solid plate. Each of the unbounded solid and MPP panels has mass per unit area $M$ and $m$, respectively. The component of the incident wavenumber vector directed parallel to the panel is $k_{z}=k \sin \varphi$. As the panel is uniform and infinite, the flexural or bending wave induced in the panel must also have the same wavenumber $k_{z}$. The incidence and reflected pressures upon the panel are given by:

$$
\begin{aligned}
& p_{i}(x<0, z)=A_{1} e^{-j k x \cos \varphi-j k z \sin \varphi} \\
& p_{r}(x<0, z)=B_{1} e^{j k x \cos \varphi-j k z \sin \varphi}
\end{aligned}
$$

where $k=\omega / c$ and $k$ represents the acoustic wavenumber, $\omega$ is the angular velocity, and $c$ is the sound speed. Here and for the rest of the equations, time dependence $e^{j \omega t}$ is assumed implicitly. At $x=0$, the acoustic pressure acting on the incident side of the front panel can be written as:

$$
p_{1}=p_{i}(x=0)+p_{r}(x=0)=A_{1}+B_{1}
$$

Similarly to Eqs. (1) and (2), the total pressure on the other side of the front panel surface $p_{2}$ can be obtained. Note that for the analysis of propagating pressure in the $x$-direction, the term $e^{-j k z \sin \varphi}$ is a constant and this is implicit in Eq. (3) as well as for the remaining equations in this paper. The relation between the average surface particle 
velocity $v$ and the sound pressure exciting the panel can be obtained by using the Euler equation $v=-1 / j \rho \omega(\mathrm{d} p / \mathrm{d} x)$. For both surfaces of each panel, at $x=0$ for the front panel, at $x=D-l$ for the MPP, and at $x=D$ for the back panel, this gives, respectively:

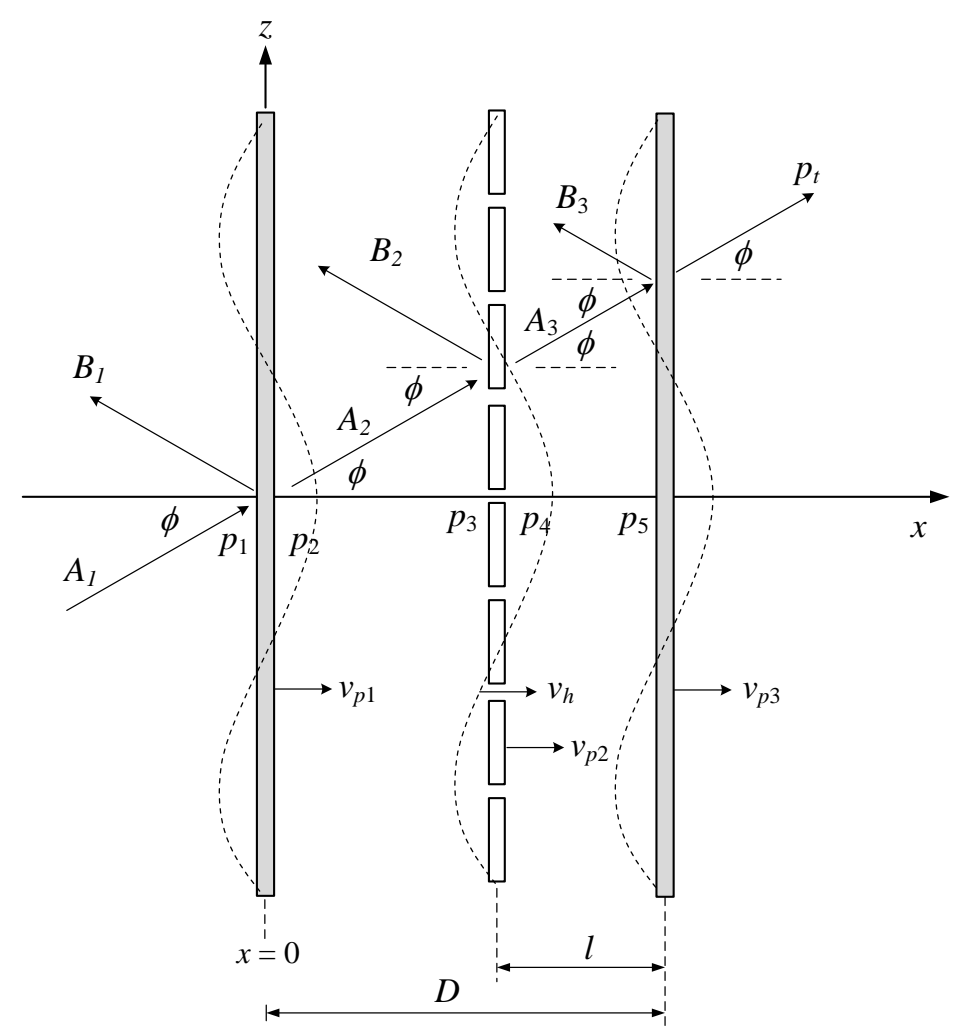

Figure 1. Schematic diagram of a DL-MPP system.

$$
\begin{gathered}
z_{f} v_{p 1}=\left(A_{1,2}-B_{1,2}\right) \cos \varphi \\
z_{f} v_{m}=\left(A_{2,3} e^{-j k(D-l) \cos \varphi}-B_{2,3} e^{j k(D-l) \cos \varphi}\right) \cos \varphi \\
z_{f} v_{p 3}=\left(A_{3} e^{-j k D \cos \varphi}-B_{2} e^{j k D \cos \varphi}\right) \cos \varphi
\end{gathered}
$$

and

$$
p_{t}=z_{f} v_{p 3} \cos \varphi
$$

where $v_{p}$ is the velocity of the panel, $v_{m}$ is the mean particle velocity over the MPP surface, and $z_{f}=\rho c$ is the acoustic impedance of air, where $\rho$ is the air density. Note that for the solid plate, the mean particle velocity on its surface is equal to the velocity of the panel $v=v_{p}$. This is valid for light fluid such as air, but not for heavy media such as water. For convenience, the distance between the panel is assumed much smaller compared with the acoustic wavelength $(k d \ll 1)$. The cavity pressures can therefore be assumed uniform between each gap:

$$
\begin{aligned}
& p_{2} \approx p_{3}=A_{2}+B_{2}=p_{b} \\
& p 4 \approx p_{5}=A_{3}+B_{3}=p_{c}
\end{aligned}
$$


Eq. (5) can be expanded to:

$$
z_{f} v_{m}=\left(A_{2,3}[\cos k(D-l)-j \sin k(D-l)]-B_{2,3}[\cos k(D-l)+j \sin k(D-l)]\right) \cos \varphi
$$

By using Eq. (8), Eq. (10) can be rewritten as:

$$
\begin{aligned}
& z_{f} v_{m}=A_{2}-B_{2}-j k(D-l) \cos \varphi p_{b} \\
& z_{f} v_{m}=A_{3}-B_{3}-j k(D-l) \cos \varphi p_{c}
\end{aligned}
$$

As the cavity pressure is uniform, the pressure between each gap can thus be expressed in terms of the front panel velocity:

$$
\begin{gathered}
p_{b}=z_{f}\left(v_{p 1}-v_{m}\right) / j k(D-l) \cos \varphi \\
p_{c}=z_{f}\left(v_{m}-v_{p 1}\right) / j k l \cos \varphi
\end{gathered}
$$

\section{Hole Impedance and Mean Particle Velocity}

As the acoustic pressure impinges on the MPP, the air particles penetrate the holes and excite the remaining solid surface of the panel. The combination between the panel velocity and particle velocity inside the holes creates the mean particle velocity given by Takahashi and Tanaka (2002):

$$
v_{m}=v_{p}(1-\sigma)+\sigma v_{h}
$$

where $v_{h}$ is the particle velocity inside the hole and $\sigma$ is the perforation ratio. The air inside the holes moves like a moving piston owing to its inertial property. At the same time, the air also interacts with the inner surface of the holes creating a friction force. These mechanisms are represented by the hole impedance. According to Maa (1975), this is given by:

$$
Z_{o}=Z_{o, R}+Z_{o, I}
$$

with

$$
\begin{gathered}
Z_{o, R}=32 v_{a} t\left[\left(1+X_{\mathrm{o}}{ }^{2} / 32\right)^{1 / 2}+\left(1.71 X_{\mathrm{o}} / 8\right) d_{\mathrm{o}} / t\right] / d_{o}{ }^{2} \\
Z_{o, I}=j \omega \rho t\left[1+\left(9+X_{\mathrm{o}}{ }^{2} / 2\right)^{-1 / 2}+0.85 d_{\mathrm{o}} / t\right]
\end{gathered}
$$

where $X_{o}=\left(d_{o} / 2\right)\left(\omega \rho / v_{a}\right)^{1 / 2}, d_{o}$ is the hole diameter, $t$ is the MPP thickness, and $v_{a}$ is the viscosity of air, i.e., $1.8 \times 10^{-5} \mathrm{Ns} / \mathrm{m}^{2}$. The resistive or real part of the impedance $Z_{o, R}$ represents the viscous effects, i.e., the interaction of the fluid particle with the wall inside the holes, while the reactive or imaginary part $Z_{o, I}$ represents the acoustic reactance from the inertia of the air. The net pressure on the surface of the panel is therefore expressed as: 


$$
Z_{o, R}\left(v_{h}-v_{p}\right)+Z_{o, I} v_{h}=\Delta p
$$

Substituting Eq. (15) into Eq. (19) gives:

$$
v_{m}=\gamma v_{p}+\sigma \Delta p / Z_{o}
$$

where $\gamma=1-\left(\sigma Z_{o, I} / Z_{o}\right)$ is the complex non-dimensional term.

\section{Sound Transmission Loss}

The equation of motion for the solid back panel is given by:

$$
z_{p 3} v_{p 3}=p_{c}-p_{t}
$$

where $z_{p 3}=-j\left(G k 4 \sin ^{4} \varphi-M \omega^{2}\right) / \omega$ is the bending mechanical impedance with $G=$ $E t^{3} / 12(1-v)$, which is the bending stiffness of the panel, $E$ is the Young's modulus, and $v$ is the Poisson's ratio. Substituting both $p_{t}$ in Eq. (7) and $p_{c}$ in Eq. (14) into Eq. (21) and then dividing both sides by $v_{p 3}$ yields the panel velocity ratio:

$$
v_{p 2} / v_{p 3}=\left[1+j k l \cos \varphi\left(1+z_{p 3} \cos \varphi / z_{f}\right)\right] /\left(\gamma+z_{p 2} / Z\right)
$$

The equation of motion for the MPP is expressed as:

$$
z_{p 2} v_{p 2}=\Delta p
$$

where $z_{p 2}=-j\left(G k 4 \sin 4 \varphi-m \omega^{2}\right) / \omega$. Substituting Eqs. (13), (14) and (20) into Eq. (23) and again dividing both sides by $v_{p 3}$ yields:

$$
v_{p 1} / v_{p 3}=\left(v_{p 2} / v_{p 3}\right)\left(j k(D-l) z_{p 2} z_{f}\right)+\left[1+j k D\left(1+z_{p 3} z_{f}\right)\right]
$$

It can be seen that the velocity ratio of the solid panels $v_{p 1} / v_{p 3}$ depends on the location of the MPP within the air gap.

The equation of motion for the front solid panel (similar to Eqs. (21) and (23)) is expressed as:

$$
z_{p 1} v_{p 1}=p_{1}-p_{b}
$$

By substituting Eqs. (3), (13) and (14) into Eq. (25), the ratio of incident to transmitted pressure is given as:

$$
\begin{aligned}
& p_{i} / p_{t}=\left(\left(v_{p 1} / v_{p 3}\right)\left[1+j k(D-l) \cos \varphi\left(1+z_{p 1} / z_{f}\right)\right]\right. \\
& \left.-\left(v_{p 2} / v_{p 3}\right)\left(\gamma+z_{p 2} / Z\right)\right) \cos \varphi / j 2 k(D-l)
\end{aligned}
$$

The case for normal incidence of acoustic loading can be obtained by setting $\varphi=0$ and the mechanical impedance of the panels can be assumed to be dominated by the mass, i.e., $z_{p 1}=z_{p 3}=j M \omega$ and $z_{p 2}=j m \omega$. 
As for plane waves, the sound power $W$ is proportional to the sound intensity $I$, which is simply a ratio of the squared magnitude sound pressure to the air impedance, i.e., $I=\left|p^{2}\right| / z_{f}$. The transmission coefficient is therefore given by $\tau=\left|p_{t} / p_{i}\right|^{2}$. For diffuse fields, this can be governed by integrating over angles of incidence, which is given by (Fahy and Gardonio, 2007):

$$
{ }_{d}={ }_{0}^{12}() \sin 2 d
$$

The transmission loss in $\mathrm{dB}$ unit is:

$$
\mathrm{STL}=10 \log _{10}(1 / \tau)
$$

\section{ANALYTICAL RESULTS}

Figure 2 shows the sound transmission loss for the case of normal incidence for partitions consisting of DL [1], triple-leaf (TL), and DLMPP for an MPP located exactly in the middle of the two panels $(l=0.5 D)$ for an air gap $D=100 \mathrm{~mm}$. The mass-airmass resonance of the DL can be seen to occur around $170 \mathrm{~Hz}$, where the STL is nearly $0 \mathrm{~dB}$. The presence of the MPP in this case does not affect this resonance because this corresponds to the gap of the double solid plates. By inserting another solid panel between the double-panel (TL), the second resonance appears at $280 \mathrm{~Hz}$, which is due to the gap between the middle and the back panel. This worsens the problem, although the STL at mid-high frequency increases significantly owing to the increase of mass within the system.

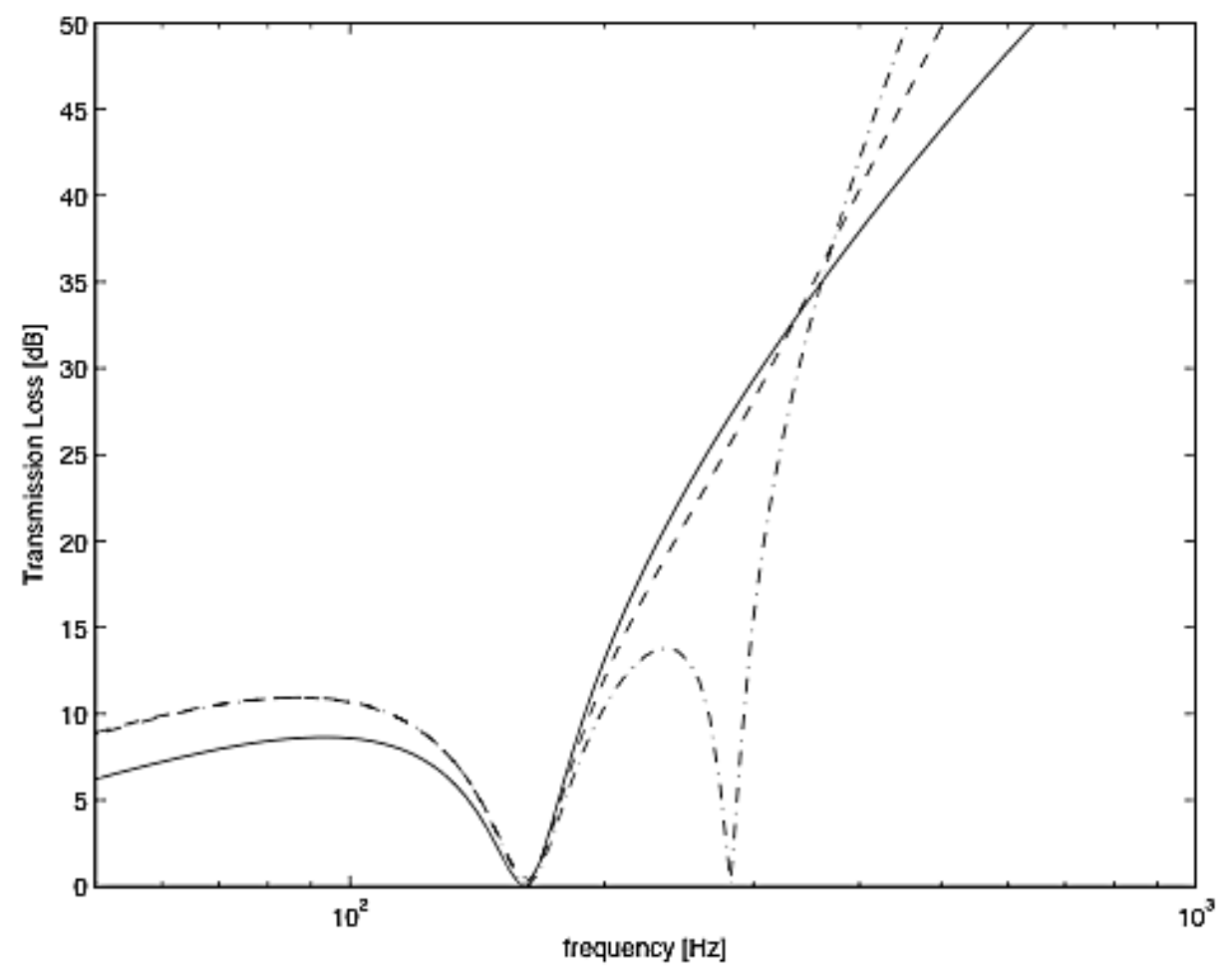

Figure 2. Sound transmission loss of DL (-), TL (- - -) and DLMPP $\left(--d_{o}=0.1 \mathrm{~mm}, \sigma=1.5 \%\right)$ subjected to normal incidence of acoustic loading. 
As the objective is to improve the STL of the conventional double-leaf partition (DL) at the mass-air-mass resonance, Figure 3 shows the STL for the DL and DLMPP for different locations of the MPP relative to the solid plate. This is plotted for the case of diffuse field incidence, which can be seen to yield a broader frequency range of resonance effects due to the summation of resonance dips at various angles of incidences. This shows that for other locations of the MPP, as the MPP shifts closer to the panel either at the front or to the back solid panel, the STL can be observed to increase at the resonance. The additional damping, due to the viscous force in the MPP holes, influences the air layer in front of the solid plate, which breaks the coupling between the solid panels and the air.

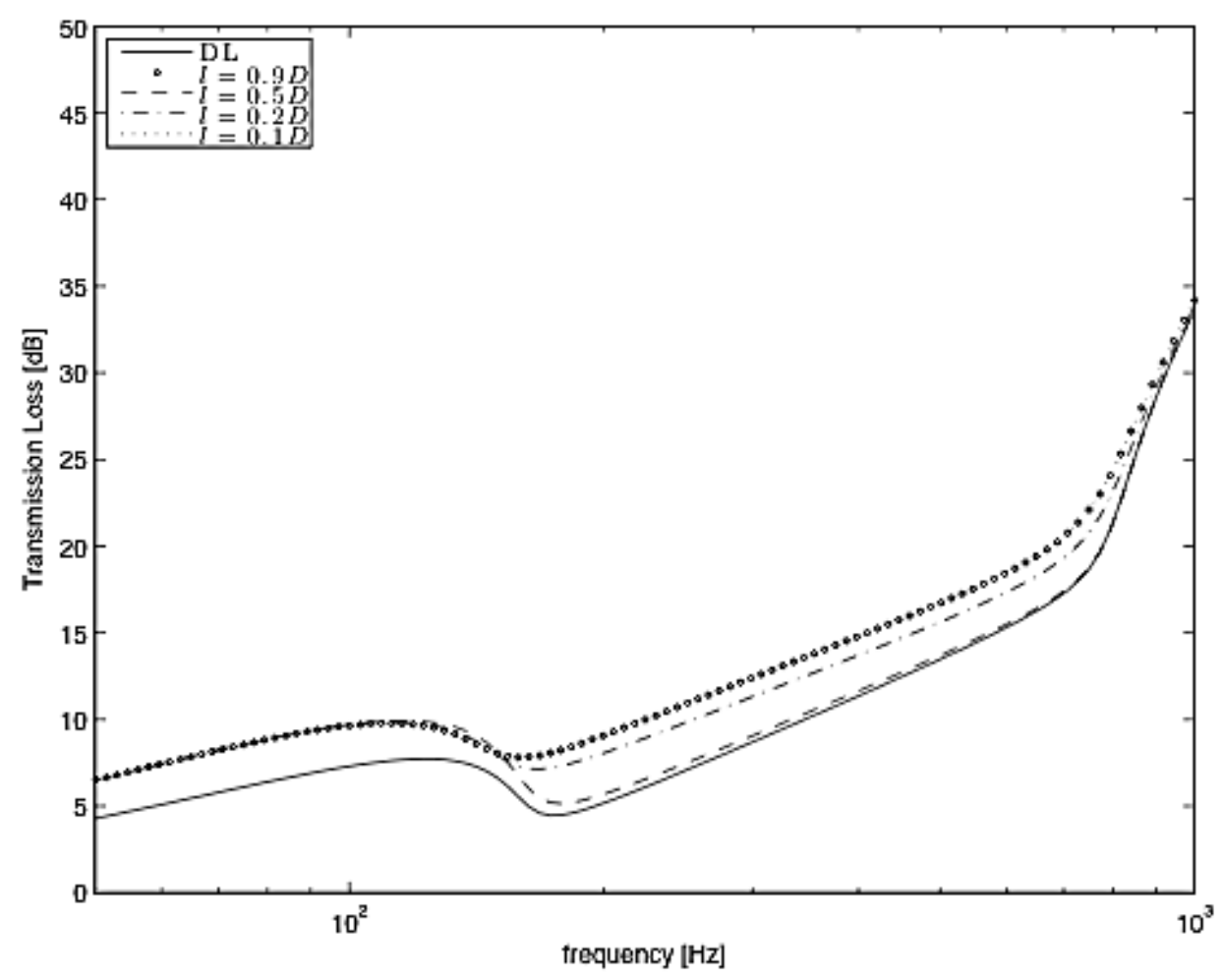

Figure 3. Sound transmission loss of DL (-) and DLMPP for different locations of MPP in the gap subjected to diffuse field incidence of acoustic loading $\left(d_{o}=0.1 \mathrm{~mm}, \sigma\right.$ $=1.5 \%$ ).

Figure 4 shows the effect of hole diameter of the MPP on the STL for a fixed MPP location at $l=0.1 D$. Around the resonance region, decreasing the hole diameter improves the STL, as this increases the domination of the real part of the hole impedance, which thus, provides more viscous force or damping to the MPP. In Figure 5 , the effect of the perforation ratio is investigated. It shows that increasing the perforation ratio does not give significant differences to the STL around the resonance. Therefore, to benefit from STL improvement at high frequency due to added mass within the system, the lowest perforation for the MPP, i.e., $\sigma=0.5 \%$ is preferred. This could also save costs for the perforated panel if the smallest hole diameter is used $\left(d_{\mathrm{o}}=0.1 \mathrm{~mm}\right)$. 


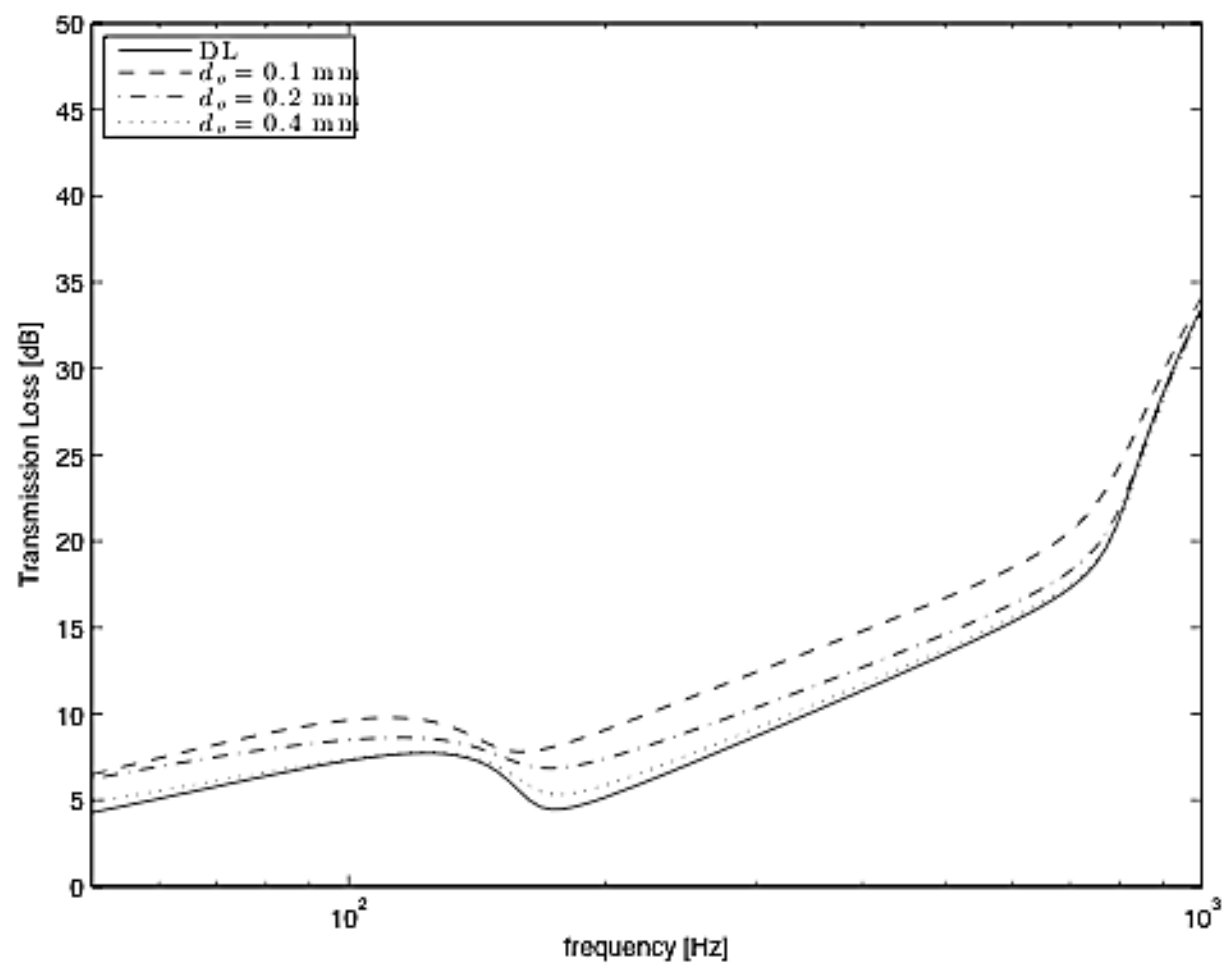

Figure 4. Sound transmission loss of DLMPP for different hole diameters $(\sigma=0.5 \%)$ subjected to diffuse field incidence $(l=0.1 D)$.

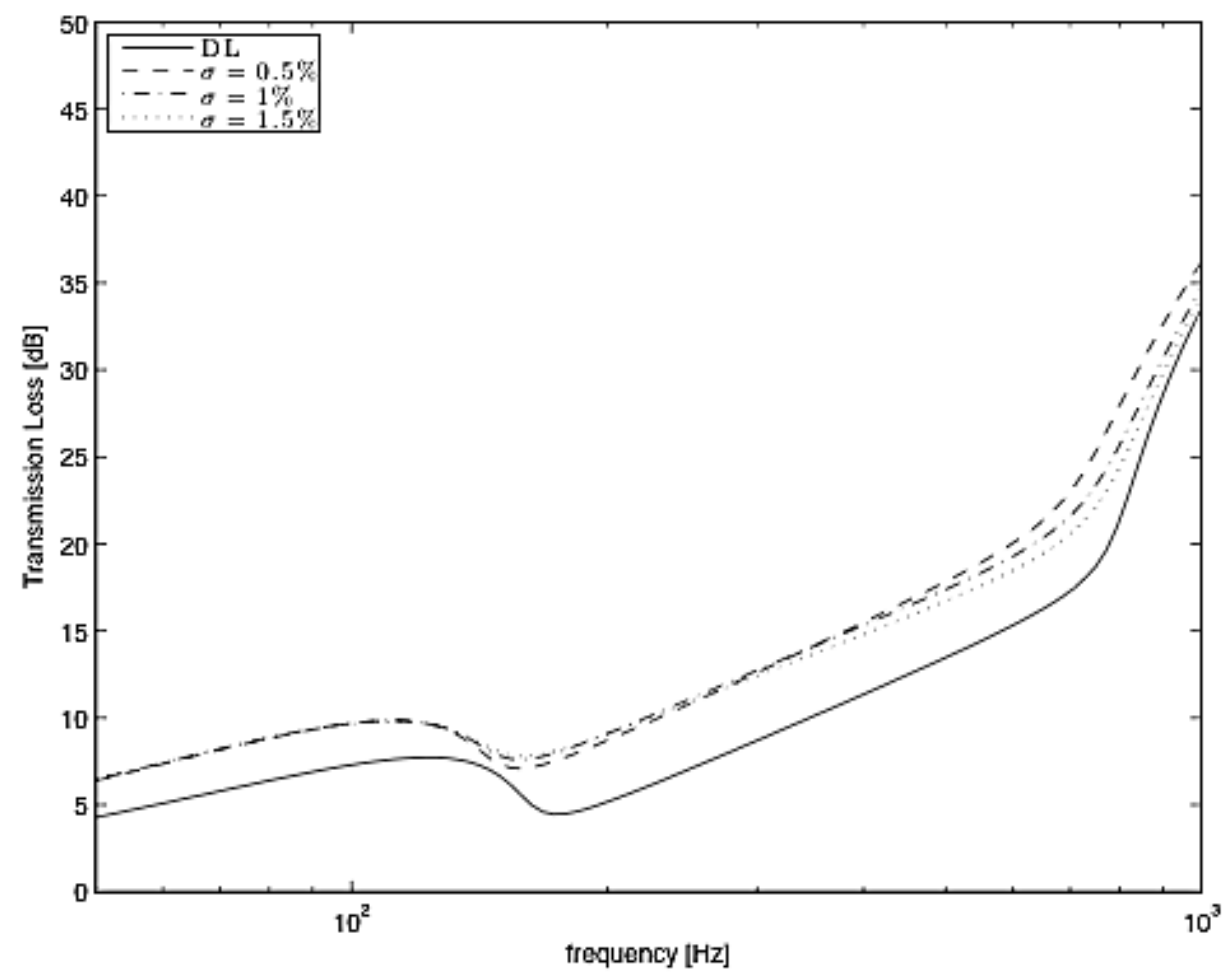

Figure 5. Sound transmission loss of DLMPP for different perforation ratios $\left(d_{o}=0.1\right.$ $\mathrm{mm})$ subjected to diffuse field incidence $(l=0.1 D)$. 
For clarity of the analysis, the level of improvement of the TL (around the resonance) can be represented by the insertion loss, i.e., the ratio of the transmitted sound power before and after the MPP insertion, which is given by:

$$
=10 \log \frac{D L}{D L M P P} \div=\mathrm{STL}_{D L M P P} \quad \mathrm{STL}_{D L}
$$

where STL DLMPP $_{\text {is }}$ the transmission loss of the DL-MPP and STL $L_{D L}$ is for the doubleleaf.

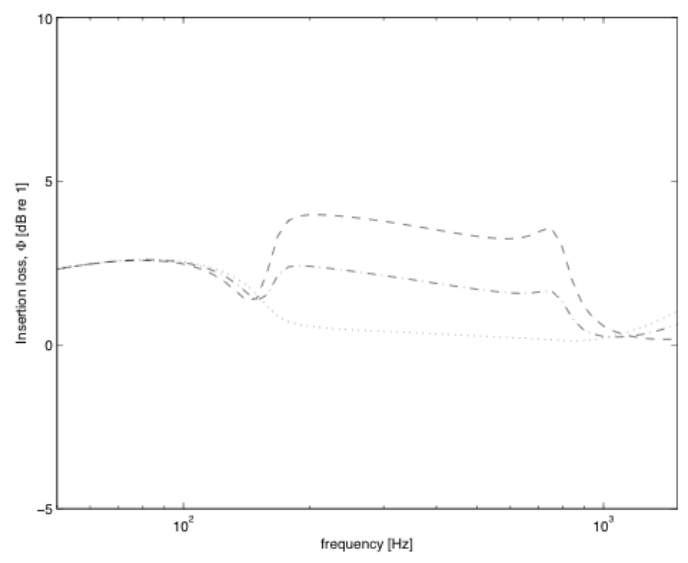

(a)

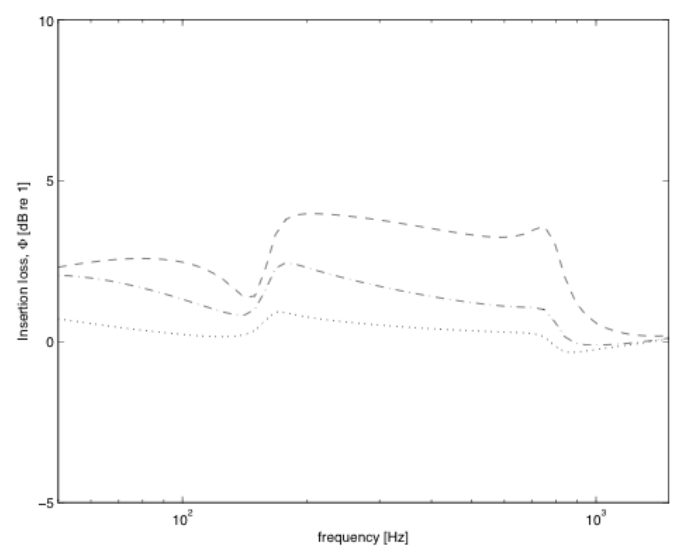

(b)

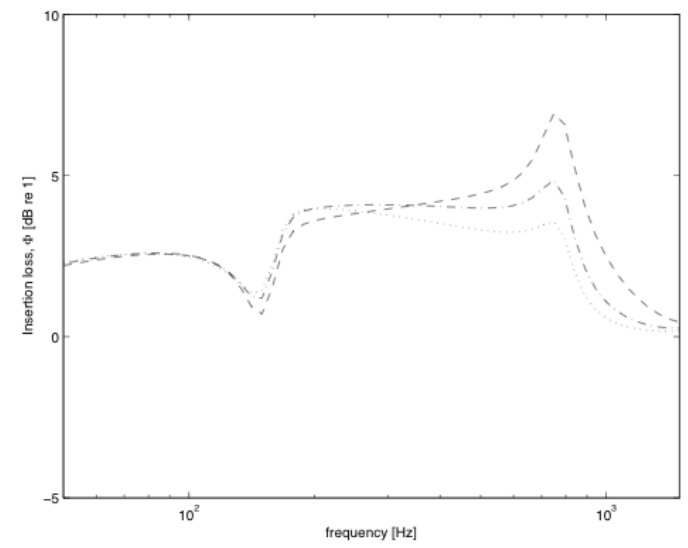

(c)

Figure 6. Insertion loss of DLMPP system with different MPP parameters: (a) locations in the gap $\left(d_{o}=0.1 \mathrm{~mm}, \sigma=1.5 \%,--l=0.1 D, \quad \times l=0.25 D, \cdots l=0.5 D\right)$, (b) hole diameters $\left(l=0.1 D, \sigma=1.5 \%,--d_{o}=0.1 \mathrm{~mm}, \quad \times d_{o}=0.2 \mathrm{~mm}, \cdots d_{o}=0.4 \mathrm{~mm}\right)$, and (c) perforation ratio $\left(l=0.1 D, d_{o}=0.1 \mathrm{~mm},--\sigma=0.5 \%, \quad \times \sigma=1 \%, \cdots \sigma=1.5 \%\right)$.

The insertion loss of the DLMPP system is presented in Figure 6. These are the results from Figures 3, 4 and 5. The improvement for DLMPP with different locations of the MPP (Figure 6(a)) can be seen to have a wider band of frequency from $200 \mathrm{~Hz}$ to $1 \mathrm{kHz}$, reaching almost $5 \mathrm{~dB}$ for $l=0.1 D$. The insertion loss for hole diameter variation is presented in Figure 6(b). It can be seen that a smaller diameter is still best for good insertion loss. Correspondingly, Figure 6(c) shows that a small perforation ratio is preferred, because varying the perforation ratio (with fixed hole diameter) gives almost no effect to the insertion loss. 


\section{CONCLUSIONS}

Models of sound transmission loss of a DLMPP system under diffuse field incidences of acoustic loading have been developed. It is found that the MPP insertion reduces the effect of resonance found in the conventional double-leaf partition. However, this is only effective when the MPP distance is less than half of the air gap and it improves as it approaches the solid plate. Reducing the size of the hole improves the STL at resonance, while changing the perforation ratio gives almost no effect on the STL performance at resonance. The model can be used for designing a multi-layer partition where problems of mass-air-mass resonance might be experienced due to a predominantly low frequency noise source.

\section{ACKNOWLEDGMENTS}

The authors gratefully acknowledge the financial support for this project by The Ministry of Higher Education Malaysia (MoHE) under Fundamental Research Grant Scheme, FRGS/2010/FKM/TK02/3-F0078.

\section{REFERENCES}

Bravo, J.M., Sinisterra, J., Uris, A., Llinaresand, L. and Estelles, H. 2002. Influence of air layers and damping layers between gypsum boards on sound transmission. Applied Acoustics, 63: 1051-1059.

Fahy, F.J. and Gardonio, P. 2006. Sound and structural vibration: radiation, transmission and response. London: Academic Press, 2nd edition.

Li, D., Zhang, X., Cheng, L. and Yu, G. 2002. Effectiveness of T-shaped acoustic resonators in low-frequency sound transmission control of a finite double-panel partition. Journal of Sound and Vibration, 329: 4740-4755.

Li, Y.Y. and Cheng, L. 2008. Mechanism of active control of sound transmission through a linked double wall system into an acoustic cavity. Applied Acoustics, 69: 614-623.

Maa, D.Y. 1975. Theory and design of microperforated panel sound absorbing constructions (in Chinese). Scientia Sinica, 18: 55-71.

Mao, Q. and Pietrzko, S. 2005. Control of sound transmission through double wall partition using optimally tuned Hemholtz resonators. Applied Acoustics, 91: 723731.

Pfretzner, J. and Cobo, P. 2006. Microperforated insertion units: An alternative strategy to design microperforated panels. Applied Acoustics, 67: 62-73.

Sakagami, K., Morimoto, M. and Koike, W. 2006. A numerical study of double-leaf microperforated panel absorbers. Applied Acoustics, 67: 609-619.

Sakagami, K., Morimoto, M., Yairi, M. and Minemura, A. 2008. A pilot study on improving the absorptivity of a thick microperforated panel absorbers. Applied Acoustics, 60: 179-182.

Takahashi, D. and Tanaka, M. 2002. Flexural vibration of perforated plates and porous elastic materials under acoustic loading. Journal of The Acoustical Society of America, 112: 1456-1464.

Tang, W.C., Cheng, H. and Ng, C.F. 1998. Low frequency sound transmission through close-fitting finite sandwich panels. Applied Acoustics, 55: 13-30. 